\title{
The Omega model: from bankruptcy to occupation times in the red
}

\author{
Hans U. Gerber $\cdot$ Elias S. W. Shiu • Hailiang Yang
}

Received: 1 March 2012/Revised: 5 June 2012/Accepted: 4 July 2012 /

Published online: 31 July 2012

(C) The Author(s) 2012. This article is published with open access at Springerlink.com

\begin{abstract}
Ruin occurs the first time when the surplus of a company or an institution is negative. In the Omega model, it is assumed that even with a negative surplus, the company can do business as usual until bankruptcy occurs. The probability of bankruptcy at a point of time only depends on the value of the negative surplus at that time. Under the assumption of Brownian motion for the surplus, the expected discounted value of a penalty at bankruptcy is determined, and hence the probability of bankruptcy. There is an intrinsic relation between the probability of no bankruptcy and an exposure random variable. In special cases, the distribution of the total time the Brownian motion spends below zero is found, and the Laplace transform of the integral of the negative part of the Brownian motion is expressed in terms of the Airy function of the first kind.
\end{abstract}

Keywords Omega model · Discounted penalty · Probability of bankruptcy · Occupation times · Airy functions

H. U. Gerber

Faculty of Business and Economics, University of Lausanne, 1015 Lausanne, Switzerland e-mail: hgerber@unil.ch

H. U. Gerber · H. Yang ( $\square)$

Department of Statistics and Actuarial Science, The University of Hong Kong, Pokfulam Road, Hong Kong, China e-mail: hlyang@hku.hk

E. S. W. Shiu

Department of Statistics and Actuarial Science, The University of Iowa, Iowa City, IA 52242-1409, USA

e-mail: elias-shiu@uiowa.edu 


\section{Introduction}

There are two parts in this note. Both are related to the Omega model that was introduced in Albrecher et al. [1]. In classical risk theory, a company goes out of business as soon as ruin occurs, that is, when the surplus is negative for the first time. In the Omega model, there is a distinction between ruin (negative surplus) and bankruptcy (going out of business). It is assumed that even with a negative surplus, the company can do business as usual and continue until bankruptcy occurs. The probability for bankruptcy is quantified by a bankruptcy rate function $\omega(x)$, where $x$ is the value of the negative surplus. The symbol for this function leads to the name Omega model. The idea of distinguishing ruin from bankruptcy comes from the impression that some companies and certain industries seem to be able to continue doing business even when they are technically ruined. This may especially be true for companies that are owned by governments or other companies.

Two of the main tasks in classical risk theory are to calculate the probability of ruin and the expectation of a discounted penalty at the time of ruin. See Gerber and Shiu [6], for example. Here, we determine the probability of bankruptcy and the expectation of a discounted penalty at the time of bankruptcy. This is done in the first part of this note (Sects. 2, 3). By assuming a Brownian motion for the surplus process, explicit results are derived.

The second part (Sects. 4, 5) of this note presents a perhaps unexpected mathematical application of the Omega model. The key for the application is formula (25). Here, the Brownian motion is assumed to have a positive drift. By considering its sample paths, various random variables are defined. Two examples are $L$, the total time of the Brownian motion below zero, and the integral of the negative part of the Brownian motion. By calculating the probability of bankruptcy for a suitable bankruptcy rate function, we can determine the Laplace transforms and hence moments of these random variables. In the special case of a constant bankruptcy rate function and the Brownian motion starting at zero, the distribution of $L$ is easily determined by identifying its Laplace transform. It is found that the probability density function of $L$ is twice a gamma probability density function (with shape parameter 1/2) minus the associated equilibrium probability density function. Some results can be viewed as limiting cases of the compound Poisson model; see Albrecher et al. [3], dos Reis [5], Loisel [9] and Zhang and $\mathrm{Wu}$ [11].

\section{Expected discounted penalty at bankruptcy}

As in Albrecher et al. [1], the surplus of a company is modeled by a Brownian motion, and the company goes out of business when bankruptcy occurs. The surplus at time $t$ is

$$
U(t)=u+\mu t+\sigma W(t), \quad t \geq 0 .
$$

Here $u$ is the initial surplus, $\mu$ is the expected gain per unit time, $\sigma^{2}$ the variance of the gain per unit time, and $\{W(t)\}$ is a standard Wiener process. The probabilistic mechanism of bankruptcy is given by a bankruptcy rate function $\omega(x) \geq 0, x \leq 0$. 
Whenever the surplus is negative and has the value $x, \omega(x) d t$ is the probability for bankruptcy within $d t$ time units. Note that the function $\omega(x)$ does not depend on other variables, such as the amount of time that the surplus has been below zero.

In analogy to Gerber and Shiu [6], we assume a bounded penalty function $w(x)$, where $x$ is now the negative surplus at the time of bankruptcy. We are interested in the function

$$
\phi(u)=E\left[e^{-\delta T} w(U(T)) I_{(T<\infty)} \mid U(0)=u\right], \quad-\infty<u<\infty,
$$

the expected present value of the penalty at bankruptcy, where $T$ denotes the time of bankruptcy. In this section, we assume a positive force of interest, i.e., $\delta>0$.

The function $\phi(u)$ is obtained as the solution of the differential equations

$$
\begin{gathered}
D \phi^{\prime \prime}(u)+\mu \phi^{\prime}(u)-[\omega(u)+\delta] \phi(u)+\omega(u) w(u)=0, \quad u<0, \\
D \phi^{\prime \prime}(u)+\mu \phi^{\prime}(u)-\delta \phi(u)=0, \quad u>0,
\end{gathered}
$$

in conjunction with the conditions that $\phi(u)$ and $\phi^{\prime}(u)$ are continuous at $u=0$, and that $\phi(u)$ is bounded for $u \rightarrow-\infty$. We have used the convenient notation

$$
D=\frac{1}{2} \sigma^{2}
$$

If we define $\omega(x)=0$ for $x>0$, Eqs. (3) and (4) can be combined as a single equation.

Because $\phi(\infty)=0$, the solution of the homogeneous differential equation (4) is

$$
\phi(u)=\phi(0) e^{-\rho u}, \quad u>0,
$$

where $-\rho$ is the negative solution of the characteristic equation associated to (4),

$$
D \xi^{2}+\mu \xi-\delta=0
$$

With $u>0$, for bankruptcy to ever occur, the surplus must first drop to 0. Formula (6) shows that the expected discounted value of a payment of 1 at the time when the surplus drops to 0 is $e^{-\rho u}$. To get more specific results, we have to make more specific assumptions about the function $\omega(x)$ and solve (3) in conjunction with the continuity conditions at $u=0$ and the boundedness condition at $-\infty$.

Equation (3) can be derived as follows. For $u<0$, we distinguish whether or not bankruptcy occurs within $d t$ time units. Thus, apart from terms of order $(d t)^{2}$ and higher,

$$
\begin{aligned}
\phi(u) & =[\omega(u) d t] w(u)+[1-\omega(u) d t] e^{-\delta d t} E[\phi(u+\mu d t+\sigma W(d t))] \\
& =[\omega(u) d t] w(u)+[1-\omega(u) d t][1-\delta d t]\left[\phi(u)+\mu \phi^{\prime}(u) d t+D \phi^{\prime \prime}(u) d t\right],
\end{aligned}
$$

which leads to (3) after simplification.

Remark 2.1 Formula (6) still holds if the surplus process is a Levy process that is skip-free downwards. The constant $\rho>0$ is determined by the condition that $\left\{e^{-\delta t+\rho U(t)}\right\}$ is a martingale. 


\section{Constant bankruptcy rates}

Typically, the bankruptcy rate function is a decreasing function, for example, from $\infty$ to 0 as $x$ goes from $-\infty$ to 0 . In contrast to this, we assume in this section that $\omega(x)$ is constant. The positive constant is denoted as $\lambda$, i.e.,

$$
\omega(x)=\lambda, \quad x<0 .
$$

Under this assumption, bankruptcy can be explained in the framework of randomized observation periods as in Albrecher et al. [2, 3]. Let $\tau_{1}, \tau_{2}, \ldots$ be i.i.d. exponential random variables with mean $1 / \lambda$. Let $T_{k}=\tau_{1}+\cdots+\tau_{k}$ denote the $k$ th observation time, $k=1,2, \ldots$. Here bankruptcy is defined as the event that the surplus is ever negative at one of these observation times. From the memoryless property of the exponential distribution it follows that the resulting bankruptcy rate is also the constant $\lambda$, which shows the equivalence of the two definitions.

We are now ready to determine $\phi(u)$. By conditioning on $\tau_{1}$ and $U\left(\tau_{1}\right)$ and using the discounted density defined by (67) in the Appendix, we have

$$
\begin{aligned}
\phi(u) & =\int_{0}^{\infty} e^{-\delta t} f_{\tau_{1}}(t)\left[\int_{-\infty}^{\infty} \phi(u+x) f_{X(t)}(x) d x\right] d t \\
& =\int_{-\infty}^{\infty} \phi(u+x) f_{X(\tau)}^{\delta}(x) d x \\
& =\int_{-\infty}^{-u} w(u+x) f_{X(\tau)}^{\delta}(x) d x+\int_{-u}^{\infty} \phi(u+x) f_{X(\tau)}^{\delta}(x) d x
\end{aligned}
$$

Applying (68) to (8) for $u=0$ yields

$$
\phi(0)=\kappa \int_{-\infty}^{0} w(x) e^{-\alpha x} d x+\kappa \int_{0}^{\infty} \phi(x) e^{-\beta x} d x .
$$

Applying (6) to the last integral in (9) and rearranging, we obtain

$$
\begin{aligned}
\phi(0) & =\frac{\kappa \int_{-\infty}^{0} w(x) e^{-\alpha x} d x}{1-\kappa \int_{0}^{\infty} e^{-(\rho+\beta) x} d x} \\
& =\frac{\kappa(\rho+\beta)}{\rho+\beta-\kappa} \int_{-\infty}^{0} w(x) e^{-\alpha x} d x \\
& =-(\rho+\alpha) \int_{-\infty}^{0} w(x) e^{-\alpha x} d x
\end{aligned}
$$

because of (72) of the Appendix. Together with (6), this yields $\phi(u)$ for $u \geq 0$. To obtain $\phi(u)$ for $u<0$, we return to (8) which is now 


$$
\begin{aligned}
\phi(u) & =\kappa \int_{-\infty}^{0} w(u+y) e^{-\alpha y} d y+\kappa \int_{0}^{-u} w(u+y) e^{-\beta y} d y+\kappa \int_{-u}^{\infty} \phi(u+y) e^{-\beta y} d y \\
& =\kappa \int_{-\infty}^{u} w(x) e^{\alpha(u-x)} d x+\kappa \int_{u}^{0} w(x) e^{\beta(u-x)} d x+\frac{\kappa}{\rho+\beta} \phi(0) e^{\beta u}
\end{aligned}
$$

where $\phi(0)$ is given by (10).

Let $f^{\delta}(x \mid u), x<0$, denote the discounted density of the surplus at bankruptcy given that the initial surplus is $u$. Because (10) and (11) are for arbitrary penalty functions, $f^{\delta}(x \mid u)$ is the coefficient of $w(x)$ in these formulas. Thus

$$
f^{\delta}(x \mid 0)=-(\rho+\alpha) e^{-\alpha x},
$$

from which and (6) it follows that

$$
f^{\delta}(x \mid u)=-(\rho+\alpha) e^{-\rho u-\alpha x}, \quad u \geq 0 .
$$

From (11) and (10), it follows that

$$
f^{\delta}(x \mid u)=\left\{\begin{array}{lll}
\kappa e^{\alpha(u-x)}-\kappa \frac{\rho+\alpha}{\rho+\beta} e^{\beta u-\alpha x}, & \text { if } & x<u<0, \\
\kappa e^{\beta(u-x)}-\kappa \frac{\rho+\alpha}{\rho+\beta} e^{\beta u-\alpha x}, & \text { if } & u<x<0 .
\end{array}\right.
$$

Remark 3.1 We note that (10) and (13) can be obtained as limits from Example 4.2 and formula (2.17) in Albrecher et al. [3].

Finally, let us determine $\phi(u)$ in the special case $w(x)=1$. Of course, we could simply set $w(x)=1$ in (10) and (11). Alternatively, we find $\phi(u)$ as the solution of the differential equation (3), which is now

$$
D \phi^{\prime \prime}(u)+\mu \phi^{\prime}(u)-(\lambda+\delta) \phi(u)+\lambda=0, \quad u<0,
$$

and (6), in conjunction with the smooth junction condition

$$
\phi^{\prime}(0-)=\phi^{\prime}(0+)
$$

and the boundedness condition of $\phi(u)$ for $u \rightarrow-\infty$. We note that $\alpha$ and $\beta$ are the solutions of the characteristic equation that is associated to (15). It follows from this and the boundedness condition that

$$
\phi(u)=B e^{\beta u}+\frac{\lambda}{\lambda+\delta}, \quad u \leq 0,
$$

The constant $B$ is determined by (16) and (6),

$$
\beta B=-\rho \phi(0)=-\rho\left(B+\frac{\lambda}{\lambda+\delta}\right) .
$$

Thus

$$
B=-\frac{\lambda \rho}{(\rho+\beta)(\lambda+\delta)}
$$


and hence

$$
\phi(u)=\frac{\lambda}{\lambda+\delta}\left(1-\frac{\rho}{\rho+\beta} e^{\beta u}\right), \quad u \leq 0 .
$$

In particular,

$$
\phi(0)=\frac{\lambda}{\lambda+\delta} \frac{\beta}{\rho+\beta} .
$$

Remark 3.2 If we set $w(x)=1$ in (10), we obtain

$$
\phi(0)=\frac{\rho+\alpha}{\alpha} .
$$

To reconcile this result with (21) use $D \alpha \beta=-(\lambda+\delta)$ and (73) of the Appendix.

\section{The probability of bankruptcy and the time spent below zero}

We assume that $\mu>0$. Let $\psi(u)$ denote the probability of bankruptcy,

$$
\psi(u)=\operatorname{Pr}(T<\infty \mid U(0)=u), \quad-\infty<u<\infty,
$$

which is a special case of (2). There is an intrinsic relation between $\psi(u)$ and the times that the Brownian motion (1) spends below zero.

For each sample path of $\{U(t) ; 0 \leq t<\infty\}$, the exposure is the random variable

$$
\mathcal{E}=\int_{0}^{\infty} \omega(U(t)) d t
$$

recall that $\omega(x)=0$ if $x>0$. We can interpret $\mathcal{E}$ as a weighted length of time that the Brownian motion spends below zero. For a given sample path of $\{U(t) ; 0 \leq t<\infty\}$, the conditional probability for no bankruptcy is $e^{-\mathcal{E}}$. From the law of total probability it follows that

$$
1-\psi(u)=E\left[e^{-\mathcal{E}}\right]
$$

For the remainder of this section, we shall consider the special case of a constant bankruptcy rate $\lambda$ as in Sect. 3 . We shall continue the discussion of the general case in Sect. 5.

With $\omega(x)=\lambda$ for $x<0$, (25) becomes

$$
1-\psi(u)=E\left[e^{-\lambda L}\right]
$$

where $L$ is the total length of time the Brownian motion spends below zero. That is, $1-\psi(u)$ is the Laplace transform of $L$ for $U(0)=u$. Because $\delta=0, \rho$ is the "adjustment coefficient" $R$ in ruin theory,

$$
\rho=R=\mu / D
$$


Also

$$
\beta=\frac{-\mu+\sqrt{\mu^{2}+4 \lambda D}}{2 D}=\frac{R}{2}[-1+\sqrt{1+\lambda / b}],
$$

with

$$
b=\frac{\mu^{2}}{4 D}
$$

From (21) it follows that

$$
1-\psi(0)=\frac{R}{R+\beta}=\frac{2}{1+\sqrt{1+\lambda / b}} .
$$

This expression is the Laplace transform of $L$ for $u=0$. Taking its derivative at $\lambda=0$, we see that

$$
E[L]=\frac{1}{4 b}=\frac{D}{\mu^{2}}
$$

as a first result. For the inversion, we rewrite the right hand side of (30) as

$$
2 \frac{1}{\sqrt{1+\lambda / b}}-2 b \frac{1-\frac{1}{\sqrt{1+\lambda / b}}}{\lambda} .
$$

Note that $\ell(\lambda)=1 / \sqrt{1+\lambda / b}$ is the Laplace transform of

$$
g(t)=\frac{b^{1 / 2}}{\Gamma\left(\frac{1}{2}\right)} t^{\frac{1}{2}-1} e^{-b t}=\sqrt{\frac{b}{\pi t}} e^{-b t}, \quad t>0,
$$

the gamma pdf with shape parameter $1 / 2$ and scale parameter $b$, and that $[1-\ell(\lambda)] / \lambda$ is the Laplace transform of $1-G(t)$, where $G(t)$ is the corresponding cdf. Thus it follows from (32) that the pdf of $L$ for $u=0$ is

$$
2 g(t)-2 b[1-G(t)], \quad t>0 .
$$

Note that because $1 /(2 b)$ is the mean of $g(t)$, the function $2 b[1-G(t)], t>0$, is a probability density function; it is known as the equilibrium probability density function associated to $g(t)$ [8, p. 282].

Consider now the case $u>0$. By (26), (6) with $\delta=0$, and (30), the Laplace transform of $L$ is

$$
\begin{aligned}
1-\psi(u) & =1-e^{-R u}+e^{-R u}[1-\psi(0)] \\
& =1-e^{-R u}+e^{-R u} \frac{2}{1+\sqrt{1+\lambda / b}} .
\end{aligned}
$$

Hence, the distribution of $L$ is of mixed type: it has a point mass of $1-e^{-R u}$ at 0 , and a density

$$
e^{-R u}\{2 g(t)-2 b[1-G(t)]\} \quad \text { for } t>0 \text {. }
$$


This result has the following probabilistic interpretation: with probability $1-e^{-R u}$, the Brownian motion will never drop to zero (in which case $L$ has the value 0 ), and with probability $e^{-R u}$ the Brownian motion will reach zero. In this case, the conditional pdf of $L$ is given by (34). Formula (36) can be shown to match the top formula on page 258 of Borodin and Salminen [4] by noting that

$$
1-G(t)=\operatorname{Erfc}(\sqrt{b t}),
$$

where $\operatorname{Erfc}(x)$ denotes the complementary error function.

Finally, we consider the case $u<0$. By (26) and (20) with $\delta=0$, the Laplace transform of $L$ is

$$
1-\psi(u)=e^{u \beta} \frac{R}{\beta+R},
$$

which can be evaluated using (28) and (30). Formula (38) shows that

$$
E\left[e^{-\lambda L}\right]=E\left[e^{-\lambda L_{I}}\right] E\left[e^{-\lambda L_{I I}}\right],
$$

where $L$ is the sum of two independent random variables, $L=L_{I}+L_{I I}$.

Here $L_{I}$ is the first passage time at 0 and $L_{I I}$ is the total time spent below zero after time $L_{I}$. It is well-known that $L_{I}$ has an inverse Gaussian distribution with Laplace transform $e^{u \beta}$ and pdf

$$
\frac{-u}{2 \sqrt{\pi D}} t^{-3 / 2} \exp \left[-\frac{(\mu t+u)^{2}}{4 D t}\right], \quad t>0,
$$

and, of course, the pdf of $L_{I I}$ is given by (34). The pdf of $L$ is given by the last formula on page 257 of Borodin and Salminen [4] with the correction that a minus sign is missing in the first exponent.

Remark 4.1 Section 5 in dos Reis [5] contains results about the Laplace transform of $L$ in the case of the classical compound Poisson model. Section 4 in Zhang and $\mathrm{Wu}$ [11] has the corresponding results in the compound Poisson perturbed by diffusion model. Our (35) is contained as a special case in their (4.10).

Remark 4.2 Relation (25) holds for more general processes. Thus also here, one strategy is to determine the probability of bankruptcy $\psi(u)$ for $\omega(x)=\lambda$, in order to obtain the Laplace transform of $L$.

\section{General bankruptcy rate functions}

For an arbitrary bankruptcy function $\omega(x), x<0, \psi(u)$ satisfies the differential equation (3) with $\delta=0$ and $w(u)=1$, i.e.,

$$
D \psi^{\prime \prime}(u)+\mu \psi^{\prime}(u)-\omega(u) \psi(u)+\omega(u)=0, \quad u<0 .
$$

It follows that

$$
\psi(u)=1+C h(u), \quad u<0
$$


where $C$ is a constant and $h(u)$ is a non-trivial solution of the corresponding homogeneous differential equation

$$
D h^{\prime \prime}(u)+\mu h^{\prime}(u)-\omega(u) h(u)=0, \quad u<0,
$$

such that $h(-\infty)=0$. We note that $h(u)$ is the auxiliary function in Section 5 of Albrecher et al. [1] with $\delta=0$. In the special case $\omega(x)=\lambda, h(u)=e^{\beta u}$ (apart from a constant factor) with $\beta$ given by (28). From (41) it follows that

$$
1-\psi(u)=\frac{h(u)}{h(0)}[1-\psi(0)], \quad u \leq 0 .
$$

Then $\psi^{\prime}(0-)=\psi^{\prime}(0+)$ is the condition that

$$
-\frac{h^{\prime}(0)}{h(0)}[1-\psi(0)]=-R \psi(0),
$$

which yields

$$
\psi(0)=\frac{h^{\prime}(0)}{h^{\prime}(0)+R h(0)}
$$

and hence

$$
1-\psi(u)=\frac{R h(u)}{h^{\prime}(0)+R h(0)}, \quad u \leq 0,
$$

by (43). The exposure can be decomposed as $\mathcal{E}=\mathcal{E}_{I}+\mathcal{E}_{I I}$. Here $\mathcal{E}_{I}$ is the exposure until the first passage time at 0 , and $\mathcal{E}_{I I}$ is the exposure thereafter. Because $\mathcal{E}_{I}$ and $\mathcal{E}_{I I}$ are independent, we have

$$
E\left[e^{-\mathcal{E}}\right]=E\left[e^{-\mathcal{E}_{I}}\right] E\left[e^{-\mathcal{E}_{I I}}\right] .
$$

This is precisely the interpretation of (43). It generalizes (39).

Remark 5.1 One might wonder how the probability of bankruptcy compares to the probability of ruin. The question makes sense for $u \geq 0$, where the probabilities are $\psi(0) e^{-R u}$ and $e^{-R u}$, respectively. Hence, their ratio is $\psi(0)$, independently of $u$. See also (6).

Now we consider two special cases. First we assume that the bankruptcy function is piecewise constant. Suppose that

$$
c_{0}=-\infty<c_{1}<\cdots<c_{n-1}<c_{n}=0
$$

and

$$
\omega(x)=\lambda_{k}, \quad \text { if } c_{k-1}<x<c_{k}, k=1, \ldots, n .
$$

Let $L_{k}$ denote the total time spent between the levels $c_{k-1}$ and $c_{k}$. Then $\mathcal{E}=$ $\sum_{k=1}^{n} \lambda_{k} L_{k}$ and (25) states that 


$$
1-\psi(u)=E\left[\exp \left(-\sum_{1}^{n} \lambda_{k} L_{k}\right)\right]
$$

Note that the expression on the right hand side is the joint Laplace transform of the random variables $L_{1}, \ldots, L_{n}$.

To determine $\psi(u)$, observe that

$$
D h^{\prime \prime}(u)+\mu h^{\prime}(u)-\lambda_{k} h(u)=0, \quad c_{k-1}<u<c_{k} .
$$

It follows that

$$
h(u)=A_{k} e^{\alpha_{k} u}+B_{k} e^{\beta_{k} u}, \quad c_{k-1}<u<c_{k},
$$

where $\alpha_{k}<0$ and $\beta_{k}>0$ are the solutions of

$$
D \xi^{2}+\mu \xi-\lambda_{k}=0 .
$$

Note that $A_{1}=0$ (because $h(-\infty)=0$ ), and we may set $B_{1}=1$. Continuity of $h(u)$ and $h^{\prime}(u)$ at $u=c_{k}$ implies that

$$
\begin{gathered}
A_{k} e^{\alpha_{k} c_{k}}+B_{k} e^{\beta_{k} c_{k}}=A_{k+1} e^{\alpha_{k+1} c_{k}}+B_{k+1} e^{\beta_{k+1} c_{k}}, \\
A_{k} \alpha_{k} e^{\alpha_{k} c_{k}}+B_{k} \beta_{k} e^{\beta_{k} c_{k}}=A_{k+1} \alpha_{k+1} e^{\alpha_{k+1} c_{k}}+B_{k+1} \beta_{k+1} e^{\beta_{k+1} c_{k}},
\end{gathered}
$$

$k=1, \ldots, n-1$. Thus, the $A$ 's and the $B^{\prime} s$ can be determined recursively by the formulas

$$
A_{k+1}\left(\alpha_{k+1}-\beta_{k+1}\right) e^{\alpha_{k+1} c_{k}}=A_{k}\left(\alpha_{k}-\beta_{k+1}\right) e^{\alpha_{k} c_{k}}+B_{k}\left(\beta_{k}-\beta_{k+1}\right) e^{\beta_{k} c_{k}}
$$

and

$$
B_{k+1}\left(\beta_{k+1}-\alpha_{k+1}\right) e^{\beta_{k+1} c_{k}}=A_{k}\left(\alpha_{k}-\alpha_{k+1}\right) e^{\alpha_{k} c_{k}}+B_{k}\left(\beta_{k}-\alpha_{k+1}\right) e^{\beta_{k} c_{k}} .
$$

Then (48) for $u=0$ is given by (45) with

$$
\begin{aligned}
h(0) & =A_{n}+B_{n}, \\
h^{\prime}(0) & =A_{n} \alpha_{n}+B_{n} \beta_{n} .
\end{aligned}
$$

There is little hope that the joint Laplace transform (48) can be inverted. But, in some cases, it might be possible to obtain moments of the joint distribution of $L_{1}, \ldots, L_{n}$.

For the second special case, we assume that $\omega(x)=-\lambda x, x<0$, for some $\lambda>0$. Thus

$$
\mathcal{E}=\lambda \int_{0}^{\infty} U(t) \_d t
$$

where $U(t)_{-}=\max (-U(t), 0)$, and (25) gives the Laplace transform of $\int_{0}^{\infty} U(t) \_d t$. Note that this random variable may be more meaningful than $L$.

By a transformation of variables, (42) with $\omega(x)=-\lambda x$ can be brought to the form that characterizes the Airy functions. Thus we find that 


$$
h(u)=e^{-\frac{R u}{2}} A i\left(\left(\frac{\lambda}{D}\right)^{1 / 3}\left(-u+\frac{\mu^{2}}{4 D \lambda}\right)\right)
$$

or a constant multiple thereof. Here $A i(x)$ denotes the Airy function of the first kind, which vanishes at $\infty$. In fact, it is known that

$$
A i(x) \sim \frac{e^{-\frac{2}{3} x^{3 / 2}}}{2 \sqrt{\pi} x^{1 / 4}} \quad \text { for } x \rightarrow \infty .
$$

See, for example, page 214 of Polyanin and Zaitsev [10]. It follows that

$$
A i^{\prime}(x) \sim-\sqrt{x} A i(x) \text { for } x \rightarrow \infty .
$$

Now (46) can be evaluated with

$$
h(0)=A i\left(z^{2}\right)
$$

where

$$
z=\sqrt{\left(\frac{\lambda}{D}\right)^{1 / 3} \frac{\mu^{2}}{4 D \lambda}}=\frac{R}{2}\left(\frac{D}{\lambda}\right)^{1 / 3}
$$

and

$$
h^{\prime}(0)=-\frac{R}{2} h(0)-\left(\frac{\lambda}{D}\right)^{1 / 3} A i^{\prime}\left(z^{2}\right) .
$$

Thus, for $u=0$, the Laplace transform of the random variable $\int_{0}^{\infty} U(t) \_d t$, with respect to $\lambda$, is

$$
\frac{2 R A i\left(z^{2}\right)}{\operatorname{RAi}\left(z^{2}\right)-2\left(\frac{\lambda}{D}\right)^{1 / 3} A i^{\prime}\left(z^{2}\right)} .
$$

To check that the limit of this expression is 1 for $\lambda \rightarrow 0$, we can use (52).

To obtain the expectation of $\int_{0}^{\infty} U(t)_{-} d t$, we calculated the derivative of (56) with respect to $\lambda$, and determined its limit for $\lambda \rightarrow 0$ using Mathematica. This leads to the following result:

$$
E\left[\int_{0}^{\infty} U(t)_{-} d t\right]=\frac{D^{2}}{\mu^{3}} .
$$

It is interesting to compare this result with (31). Now, consider $U(0)=u \geq 0$. Because $e^{-R u}$ is the probability that the Brownian motion $\{U(t)\}$ will drop to level 0 , we can generalize (31) and (57) as

$$
E[L]=\frac{D}{\mu^{2}} e^{-R u}=\frac{e^{-R u}}{D R^{2}}, \quad u \geq 0,
$$

and 


$$
E\left[\int_{0}^{\infty} U(t)_{-} d t\right]=\frac{D^{2}}{\mu^{3}} e^{-R u}=\frac{e^{-R u}}{D R^{3}}, \quad u \geq 0,
$$

respectively, where $R$ is defined by (27). Note that the RHS of (58) is the negative of the derivative of the RHS of (59) with respect to $u$; this is an illustration of Theorem 1 in Loisel [9].

Remark 5.2 Formulas (58) and (59) can be verified with Theorem 7 in Loisel [9]. Consider

$$
U(t)=u+c t-S(t)
$$

where $\{S(t)\}$ is a compound Poisson process with Poisson parameter $v$ and exponential claim size distribution with mean $m$. Then, Loisel's results are

$$
E[L]=\frac{(1-m R) e^{-R u}}{c m R^{2}}, \quad u \geq 0
$$

and

$$
E\left[\int_{0}^{\infty} U(t) \_d t\right]=\frac{(1-m R) e^{-R u}}{c m R^{3}}, \quad u \geq 0,
$$

where the adjustment coefficient $R$ is given by

$$
R=\frac{c-v m}{c m} .
$$

Note that the numerator in (61) and (62) is the probability of ruin; so is the numerator on the RHS of (58) and (59). In the limit $c \rightarrow \infty, v \rightarrow \infty, m \rightarrow 0$ such that

$$
c-m v=\mu,
$$

and

$$
v m^{2}=D
$$

the process (60) tends to (1). Because

$$
\mathrm{cm}^{2} \rightarrow D,
$$

the RHS of (63) tends to the RHS of (27). Hence, (61) and (62) yield (58) and (59), respectively.

Acknowledgments We acknowledge with thanks the support from the Principal Financial Group Foundation, Research Grants Council of the Hong Kong Special Administrative Region (project No. HKU 706611P), and Society of Actuaries' Centers of Actuarial Excellence Research Grant. We are grateful to Eric Cheung, You Beng Koh, and two anonymous referees for their insightful comments. 
Open Access This article is distributed under the terms of the Creative Commons Attribution License which permits any use, distribution, and reproduction in any medium, provided the original author(s) and the source are credited.

\section{Appendix: Exponential stopping of Brownian motion}

Let $X(t)=\mu t+\sigma W(t), \quad t \geq 0$, where $\{W(t)\}$ is a standard Wiener process. Let $f_{X(t)}(x)$ denote the probability density function of $X(t)$. For an independent random variable $\tau$ with exponential distribution (parameter $\lambda>0$ ) and a force of interest $\delta>0$, we consider the discounted density

$$
f_{X(\tau)}^{\delta}(x)=\lambda \int_{0}^{\infty} e^{-(\lambda+\delta) t} f_{X(t)}(x) d t .
$$

It is known that

$$
f_{X(\tau)}^{\delta}(x)= \begin{cases}\kappa e^{-\alpha x}, & \text { if } \quad x \leq 0 \\ \kappa e^{-\beta x}, & \text { if } \quad x>0\end{cases}
$$

where $\alpha<0$ and $\beta>0$ are the two solutions of the quadratic equation

$$
D \xi^{2}+\mu \xi-(\lambda+\delta)=0,
$$

with $D=\frac{1}{2} \sigma^{2}$ and

$$
\kappa=\frac{\lambda}{D(\beta-\alpha)} .
$$

For a short proof of (68), consider the two-sided Laplace transform of (67):

$$
\begin{aligned}
\int_{-\infty}^{\infty} e^{-z x} f_{X(\tau)}^{\delta}(x) d x & =\lambda \int_{0}^{\infty} e^{-(\lambda+\delta) t-\mu t z+D t z^{2}} d t \\
& =\frac{\lambda}{\lambda+\delta+\mu z-D z^{2}} \\
& =\frac{\kappa}{\beta+z}-\frac{\kappa}{\alpha+z}
\end{aligned}
$$

for $-\beta<z<-\alpha$. This is indeed the two-sided Laplace transform of (68). There is an alternative expression for $\kappa$ :

$$
\kappa=\frac{-(\rho+\alpha)(\rho+\beta)}{\beta-\alpha},
$$

where $-\rho$ is the negative solution of (7). Formulas (70) and (72) are equivalent because 


$$
-(\rho+\alpha)(\rho+\beta)=\frac{\lambda}{D} .
$$

For $\delta=0$, (68) can be found as formula 2.1.0.5 on page 250 of Borodin and Salminen [4]. Furthermore, we note that (68) can be obtained as a limiting result from the unnumbered formula on page 655 of Albrecher et al. [2], or from the first formula in Example 4.1 of Albrecher et al. [3]. Results more general than (68) and their actuarial and financial applications can be found in Gerber et al. [7].

\section{References}

1. Albrecher H, Gerber HU, Shiu ESW (2011) The optimal dividend barrier in the Gamma-Omega model. Eur Actuar J 1:43-55

2. Albrecher H, Cheung ECK, Thonhauser S (2011) Randomized observation periods for the compound Poisson risk model: dividends. ASTIN Bull 41(2):645-672

3. Albrecher H, Cheung ECK, Thonhauser S (2012) Randomized observation periods for the compound Poisson risk model: the discounted penalty function. Scand Actuar J (in press)

4. Borodin AN, Salminen P (2002) Handbook of Brownian motion-facts and formulae, 2nd edn. Birkhauser, Basel

5. Dos Reis AE (1993) How long is the surplus below zero? Insurance: Math Econ 12:23-38

6. Gerber HU, Shiu ESW (1998) On the time value of ruin. N Am Actuar J 2(1):48-72; Discussions 72-78

7. Gerber HU, Shiu ESW, Yang H (2012) Valuing equity-linked death benefits and other contingent options: a discounted density approach. Insurance: Math Econ 51(1):73-92

8. Klugman, SA, Panjer, HH, Willmot, GE (2008) Loss models: from data to decisions, 3rd edn. Wiley, Hoboken

9. Loisel S (2005) Differentiation of some functionals of risk processes, and optimal reserve allocation. J Appl Probab 42:379-392

10. Polyanin AD, Zaitsev VF (2003) Handbook of exact solutions for ordinary differential equations, 2nd edn. Chapman \& Hall/CRC, Boca Raton

11. Zhang C, Wu R (2002) Total duration of negative surplus for the compound Poisson process that is perturbed by diffusion. J Appl Probab 39:517-532 